\title{
Mapping the potential distribution of the schistosomiasis intermediate host Biomphalaria straminea in China
}

\author{
Jingyu Fan, Xuejiao Yuan, Menglin Wang, Gengping Zhu \\ Tianjin Key Laboratory of Animal and Plant Resistance, College of Life Sciences, Tianjin Normal \\ University, Tianjin, China
}

\begin{abstract}
Biomphalaria straminea is native to Brazil but has established a population in Guangdong Province, China. Its potential of expanding and transmitting Schistosoma mansoni is of great concern. We seek to map $S$. mansoni transmission potential by predicting ecological dimensions and potential distributions of $B$. straminea using state-of-the-art ecological niche model approaches. Two environmental datasets were selected to fit individual and ensemble niche models, together with the evaluation of niche conservatism during $B$. straminea invasion in China. A small area is still occupied by the introduced population compared to that in Brazil. A vast space with suitable climate remains unfilled and might be available to $B$. straminea. Contrasting results of niche
\end{abstract}

Correspondence: Gengping Zhu, Tianjin Key Laboratory of Animal and Plant Resistance, College of Life Sciences, Tianjin Normal University, Binshuixi Road 393, Tianjin 300387, China.

Tel.: +86.022.23766394 - Fax: +86.022.23766539.

E-mail: gengpingzhu@hotmail.com

Key words: Biomphalaria straminea; Biological invasion; Transmission risk; Ecological niche modelling; Climate niche conservatism; China.

Contributions: GZ conceived the idea and lead the research, JF, XY, MW collected the data and performed the analysis, all author contributed to the writing.

Conflict of interest: the authors declare no potential conflict of interest.

Funding: this work was partially supported by Tianjin 131 Creative Talents Cultivation Project (135305JF09) and National Natural Science Foundation of China (31870523).

See online Appendix for additional Figures.

Received for publication: 5 July 2018.

Revision received: 30 August 2018.

Accepted for publication: 1 October 2018.

CC Copyright J. Fan et al., 2018

Licensee PAGEPress, Italy

Geospatial Health 2018; 13:723

doi:10.4081/gh.2018.723

This article is distributed under the terms of the Creative Commons Attribution Noncommercial License (CC BY-NC 4.0) which permits any noncommercial use, distribution, and reproduction in any medium, provided the original author(s) and source are credited. conservatism evaluation were attained based on the two environmental datasets. The coastal areas in southern China, together with south-western Yunnan and southern Hainan and Taiwan were consistent supported by multiple model approaches, showing high climate suitability for B. straminea. Attention should be paid to the possibility of $S$. mansoni epidemic in these identified areas, as high pressure due to propagation and future climate change may further complicate conditions. Our results call for rigorous monitoring and supervising along these areas in China.

\section{Introduction}

Biological invasion represents a major threat to the sustainable development of our society (Mack et al., 2000), resulting in serious damages to environment and human health, which can eventually lead to great economic loss (Liu et al., 2011). Many strategies and actions are attempted to reduce the effect of invasive species, especially those that spread disease. Eradication is usually difficult once the invasive species has passed the bottleneck and established populations (Kolar and Lodge, 2001). The most effective way might be to prevent initial invasion or restrain future expansion rather than trying to curb outbreaks. It is therefore a priority to consider spatial deployment of efforts and resources for field management with regard to invasive species.

Predicting potential distribution of invasive species via ecological niche modelling (ENM) approaches could be useful by offering information on allocating efforts in this regard (e.g., Roura-Pascual et al., 2011; Beans et al., 2012). ENM seeks to characterize ecological requirements of species using environmental variables associated with observed data and then identify how suitable environmental space are distributed. This correlative approach is now routinely used with respect to biological invasion (Peterson et al., 2012), and increasingly used in disease ecology (Peterson et al., 2014).

Schistosoma mansoni is the parasite species responsible for schistosomiasis in tropical and subtropical areas in Africa and Latin America, especially in Brazil. One of its intermediate snail hosts, Biomphalaria straminea, is native to Brazil but has established populations in many countries in Central and North America and Asia. The first detection of B. straminea in China was in Kowloon in Hong Kong in the 1970s, where it was collected by shellfish amateurs in irrigation trenches (Fernandez, 2002). Pan et al. (1983) first reported its existence in Shenzhen in mainland China. By 2013, the snail had spread to surrounding regions of Dongguan City and Huizhou City in Guangdong Province (Figure 1) where it had established populations in freshwater habitat throughout urban and rural areas. Its further expansion and potential for transmitting S. mansoni have been much discussed. Habib et al. (2016) and Yang et al. (2018) employed the maximum 
entropy model (MaxEnt) (www.gbif.org/tool/81279/maxent) to produce risk maps for $B$. straminea in China. However, their models were either based on the population of a non-equilibrated state (i.e. the introduced population did not inhabit the entire habitable area), violating key assumption of ENM applications (Peterson et al., 2011) or employing a single-model approach without consideration of model uncertainty (Yang et al., 2018). Human impacts appeared to be an important factor for the establishment and expansion of B. straminea, Yang et al. (2018) added human factors in a niche model to predict the potential distribution but did not calculate human populations that at risk of $S$. mansoni transmission.

In this study, classical niche models based on a native equilibrium population were used to map ecological dimensions and potential distributions of $B$. straminea using multiple model approaches. In addition, climate niche conservatism during the invasion of B. straminea in China, which is another key assumption in ENM applications (Peterson et al., 2012), was tested using two environmental datasets. The aims of this study were i) to compare climate spaces occupied by native Brazilian populations and populations introduced into China; ii) to address whether the climate niche was diverged during B. straminea invasion in China; and iii) to predict its potential distribution in China using a finetuned individual MaxEnt approach and collective model ones.

\section{Materials and Methods}

\section{Input data}

Occurrence records of $B$. straminea were assembled from online databases (Global Biodiversity Information Facility (www.gbif.org/) and the published literature (e.g., Scholte et al., 2012; Habib et al., 2016; Zeng et al., 2017). These records were overlaid on the administrative maps and compared with their original descriptions to remove errors. Records lacking geographic coordinates were georeferenced using Google Maps. Spatial aggregations of distributional records were filtered at a 10 arc grid, where only a single record per raster cell was reserved for model calibration and evaluation to minimize possible effects of spatial autocorrelation (Veloz, 2009). A total of 542 records (507 native and 35 Chinese) reflecting the present-day distribution of $B$. straminea were retained for analysis.

Bioclimatic variables representing annual trends, extreme conditions of temperature and precipitation according to Hijmans et al. (2005) were considered. Variables combining temperature and precipitation were excluded because they displayed artificial discontinuities among adjacent grid cells in some areas (Escobar et al., 2014). Two environmental datasets were selected based on their ecological relevance and independence from other variables. Dataset A summarized the aspects of temperature, and precipitation and had a Pearson correlation value of $<0.85$. It included mean diurnal range (Bio2), temperature seasonality (Bio4), maximum temperature of warmest month (Bio5), mean temperature of coldest quarter (Bio11), annual precipitation (Bio12), precipitation seasonality (Bio15) and precipitation of driest quarter (Bio17).

The snail mainly lives in fresh water in tropical areas but grows fast and adapts to diverse environments well. We assume that annual mean and extreme values of temperature and precipitation might be more important than others in limiting snail distribution. Therefore, a dataset B was made to reflect primary relevance in imposing constraints upon snail distribution, including annual mean temperature (Bio1), Bio5, minimum temperature of coldest month (Bio6), Bio12, precipitation of wettest month (Bio13), precipitation of driest month (Bio14) and Bio15. All analyses were based on the above two datasets at a spatial resolution of 2.5 '.

\section{Climate space comparison}

Climate spaces occupied by native Brazilian and introduced Chinese populations were compared and mapped in reduced dimensions using principle component analysis (PCA) using NicheA, version 3.0 according to Qiao et al. (2015). NicheA is an open-source platform that can be used to simulate ecological niches (Qiao et al., 2015) it assumes that fundamental species niches are convex in shape as suggested by available evidence (e.g., Soberón and Nakamura, 2009). Niches therefore can be operated as minimum volume ellipsoids (Van Aelst and Rousseeuw, 2009). NicheA can quantify similarity among multiple niches in terms of overlap in n-dimensional environmental spaces (Qiao et al., 2015). Here, we mapped ecological dimensions for $B$. straminea by comparing climate spaces occupied by native and introduced populations in three reduced dimensions based on the two datasets mentioned above.

Climate niche conservatism during the invasion of $B$. straminea was investigated. We used ecospat of the R package ( $\mathrm{R}$ Core Team, 2015), a statistical software supporting spatial analyses and modelling of species niches and distributions (Cola et al., 2017), to test whether the snail's climate niche was diverged in environmental spaces. This programme uses kernel density to fit species distribution points and environmental variables associated with geographical region to test niche conservatism. Climate niche overlaps between native and introduced ranges were measured by the first two axes of PCA (Broennimann et al., 2012). Schoener's $D$, which varies between 0 (no overlap at all) and 1 (complete overlap) according to Petitpierre et al., (2012) was used to measure the overall match between the two niches.

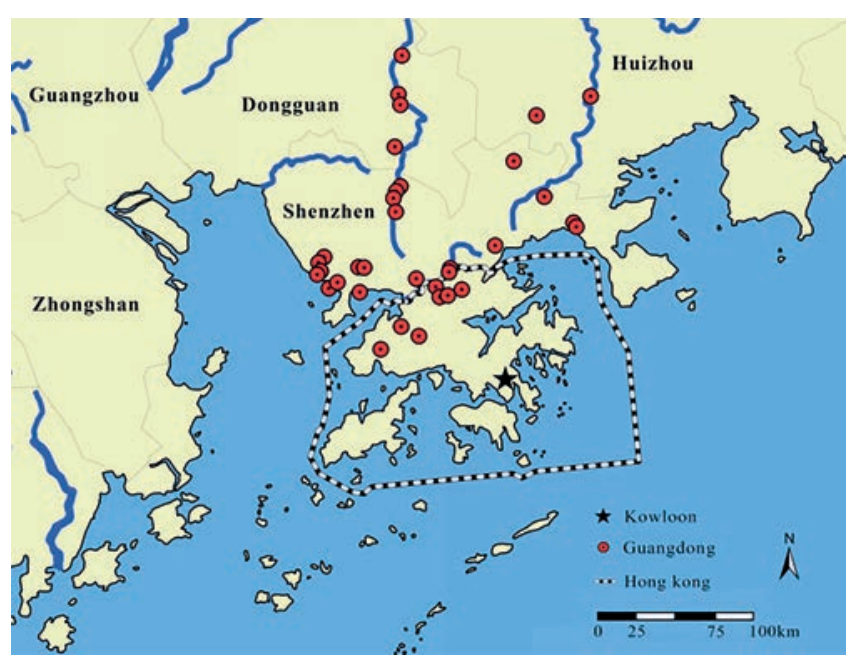

Figure 1. Map showing distribution and expansion of Biomphalaria straminea in southern China. The snail was first reported in Kowloon in Hong Kong and then expanded into surrounding areas. 
Two alternative hypotheses of equivalency and similarity in statistical tests were used to test niche conservation. The former, which was used to compare niche similarity between geographic populations, works by comparing niche overlap values generated by actual occurrence data with those generated by empirical data randomly sampled across the two regions. The latter, which was used to test whether this similarity is due to the geographical area in which they occur, works by comparing actual niche overlap with that generated using points drawn at random from the region defined as environmental background for one of the populations (Broennimann et al., 2012; Petitpierre et al., 2012).

Background environments for the comparisons of climate space and niche modelling should include areas accessible to the species (Barve et al., 2011). Here, we delimited this area by buffering known occurrences by $400 \mathrm{~km}$, which reflects a compromise between including all accessible environments (Barve et al., 2011) and covering an extent broad enough to minimize extrapolation and detect climatic differences between presence and background records (Owens et al., 2013). Climatic similarities between native and introduced populations were assessed using mobility-oriented parity (MOP) metrics for the two environmental datasets. MOP is a correction and improvement of multivariate environmental similarity surfaces (Owens et al., 2013).

\section{Ecological niche modelling calibration and evaluation}

Native models were fitted using the two datasets and then transferred across the globe to predict areas of potential distributions. $70 \%$ of native-range points were used to fit the model, the rest of them and introduced Chinese points were used for model interpolative and extrapolative evaluations respectively.

We sought to adopt both individual and collective model approaches to predict the potential distribution because each approach has its advantages and disadvantages (Zhu and Peterson, 2017). The ensemble model approach is widely used to reduce model-based uncertainty but it is not better than the individual model. Therefore, five individual commonly used modelling algorithms, i.e., generalized additive models, generalized boosted models (GBMs), generalized linear models, random forests, and MaxEnt models were developed using default parameters in biomod2 (Thuiller, 2003) implemented in R software (R Core Team, 2015) and then averaged to produce ensemble models. Eventually, two individual models were considered: MaxEnt and GBM. The former follows the principle of maximum entropy and is the most popular model algorithm, whereas the latter uses boosting to combine large numbers of relatively simple tree models, which is recommended by Jane Elith et al. (2006). Recently, Maxent based on default setting has been criticized because of its complexity and low transferability (e.g., Muscarella et al., 2014). In this study, the MaxEnt model was optimized using the ENMeval package (Muscarella et al., 2014), which provides an automated way to execute MaxEnt models across a user-specified range of regularization multiplier (RM) values and feature combination (FC). We set the RM range to $0.5-4.0$ with increments of 0.5 and used six FCs, i.e. linear $(\mathrm{L})$, quadratic $(\mathrm{Q})$, hinge $(\mathrm{H})$, product $(\mathrm{P})$ and threshold $(\mathrm{T})$ to cover a broad range of model settings. The fine-tuned MaxEnt models were made by seeking the minimum value of Akaike's information criterion (AIC) among candidate models (Muscarella et al., 2014).

Partial receiver operating characteristic (ROC) and omission error were used to evaluate model interpolation and transferability, respectively. The traditional area under the curve (AUC) approach is misleading according to Peterson et al. (2008) and JiménezValverde et al. (2012), so we adopted partial ROC approach, which considers the quality of occurrence points and weights more on omission error (Peterson et al., 2008; Zhu et al. 2017a). An AUC ratio of 1 implies that the niche model is no better than a random prediction; the larger the AUC ratio, the better the discrimination in partial ROC approach (Peterson et al., 2008). The omission

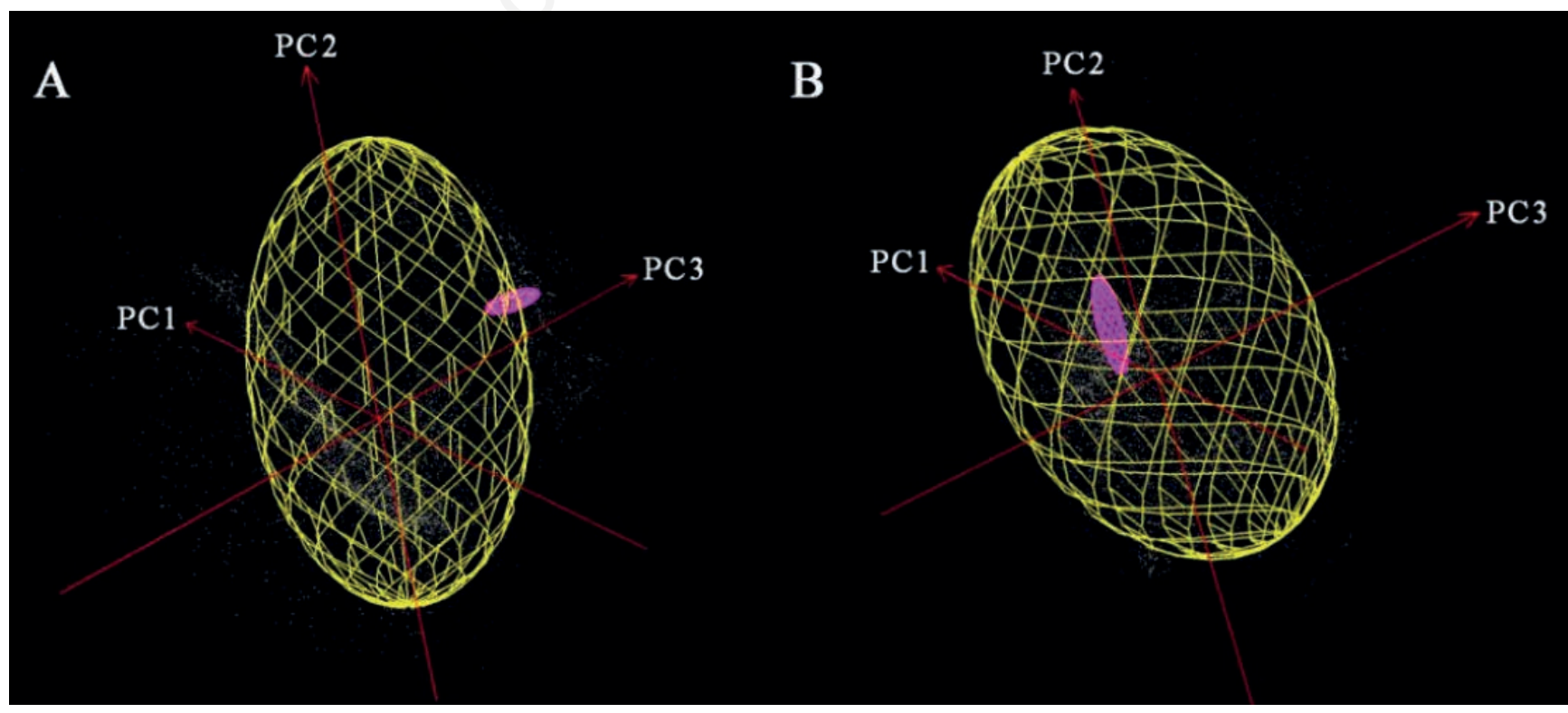

Figure 2. Climate niche spaces of Biomphalaria straminea based on two environmental datasets (datasets A and B). Yellow and pink ellipsoids represent niche spaces occupied by native and invading populations, respectively. 
error was calculated by the proportion of testing points not predicted at a threshold. Here, occurrence data in China were used to calculate omission error across the threshold spectrum of model output. Specifically, we calculated the omission error at the increment of 10 against the total 1,000 .

\section{Human risk assessment}

The human population that fell into suitable areas of $B$. straminea were estimated in four cities (i.e., Hong Kong, Dongguan, Huizhou and Shenzhen) based on our predictions. To do so, the suitability maps generated by GBM model (which showed the best performance, see results) were converted into binary prediction at the 10 percent training threshold, the population that fell into the positive predicted areas were estimated based on a global population distribution data, which were obtained from Oak Ridge National Laboratory (www.ornl.gov/sci/landscan).

\section{Results}

\section{Climate space}

PCA of the climate variables associated with snail occurrences revealed reduced dimensions that accounted for the observed distribution. In dataset A, the first three components (PC1, PC2 and PC3) explained $88.6 \%$ of the variance; PC1 was associated with mean diurnal temperature range (Bio2) and accounted for $51.5 \%$, PC2 was associated with precipitation seasonality (Bio15) and accounted for $23.3 \%$, and PC3 accounted for $13.7 \%$ and was less associated with a single dimension. In dataset $\mathrm{B}$, the first three components (PC1, PC2, and PC3) explained $88.6 \%$ of the vari- ance; PC1 was associated with annual precipitation (Bio12) and accounted for $57.7 \%$, PC2 was associated with precipitation seasonality (Bio15) and accounted for $24.8 \%$, and PC3 accounted for $11.5 \%$ and was less associated with a single dimension.

Contrasting size of ellipsoid volumes between native and introduced populations were observed in both datasets: native population occupied a large ellipsoid, whereas the introduced Chinese population occupied a relatively small ellipsoid (Figure 2). Nonetheless, the overlaps of climate spaces occupied by native and introduced populations varied between the two datasets: the two ellipsoids were partially overlapped in dataset A, but the Chinese population ellipsoid fell well into that of the native population in dataset B (Figure 2).

\section{Niche conservatism}

Climate niche conservative evaluation showed contrasting results based on the two datasets. Non-equivalency niche spaces occupied by native and introduced populations were consistently supported (dataset A: Schoener's $D=0, \mathrm{P}<0.5$; dataset $\mathrm{B}$ : Schoener's $D=0.002, \mathrm{P}<0.5$ ), as actual niche overlaps, were quite different from those of random data (Figure 3), whereas the results of similarity tests varied between the two datasets. In dataset A, the non-equivalency niche results were supported in similarity tests, as actual niche overlap fell far away from random overlaps; however this was not the case for dataset B as actual niche overlap fell inside of random overlaps (Figure 3).

\section{Model performances}

Fine-tuned parameters of MaxEnt models identified by ENMeval package were quite different from those default param-

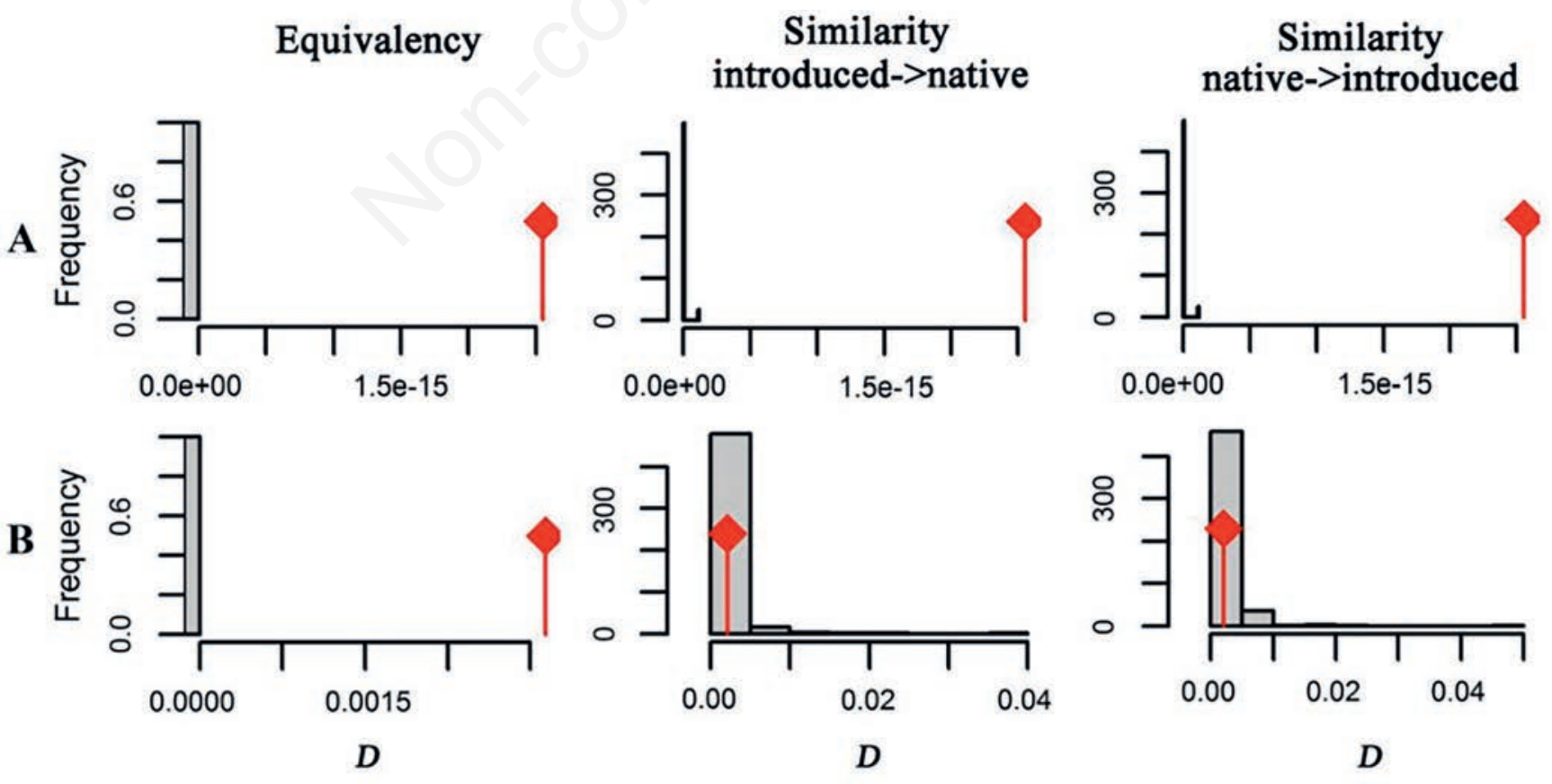

Figure 3. Histograms of niche equivalency and similarity tests between native and introduced ranges based on the two datasets. Red lines with a diamond represent the observed niche overlap, and gray bars represent simulated niche overlaps. 
eters. In dataset $\mathrm{A}$, the minimum $\mathrm{AICc}$ was attained based on the parameters of $\mathrm{RM}=4$ and Features $=\mathrm{L}, \mathrm{Q}, \mathrm{H}$ and $\mathrm{P}$. In dataset $\mathrm{B}$, the best parameters of $\mathrm{RM}=2$ and Features $=\mathrm{L}, \mathrm{Q}, \mathrm{H}$, and $\mathrm{P}$ were attained. All individual and ensemble models performed better than random predictions based on the two datasets in interpolative evaluations (AUC ratios in dataset $\mathrm{A}$ : MaxEnt $=1.92, \mathrm{GBM}=$ 1.91, ensemble $=1.94$; AUC ratios in dataset B: MaxEnt $=1.92$, $\mathrm{GBM}=1.92$, ensemble $=1.93$ ). Ensemble models generally performed better that those individual models used to generate them in native model evaluations.

When the models were transferred into China, omission errors varied across the spectrum of thresholds (Figure 4). Similar omission errors were attained in MaxEnt models between the two datasets, whereas outputs based on dataset B generally outperformed those based on dataset A in GBM and ensemble models. In GBM outputs, models based on dataset B showed lower omission errors at thresholds of 180-400 than those based on dataset A but not at thresholds less than 180 or larger than 400 . In ensemble outputs, models based on dataset B showed lower omission errors at thresholds of 100-600 but not at thresholds of less than 100 or larger than 600

\section{Model predictions}

Projecting native-range ENMs based on the two datasets onto the globe generally involved only minor amounts of strict extrapolation in dataset A, but not dataset B (MOP analysis - Appendix Figure A3). Spatial predictions by our native models showed that the suitable areas are in Central America, Sub-Saharan Africa, and Southeast Asia (Appendix Figure A1). These predictions for $B$. straminea closely matched its present-day distribution, i.e. mostly tropical and subtropical areas. Predictions of collective models were generally consistent between the two datasets, but they were more conservative than those based on individual models (Appendix Figure A1).

In China, all models showed a trend of suitability descending from southern coastal areas to the North (Figure 5; Appendix Figure A2). Areas of high suitability consistently supported by present models included coastal areas in southern China, south-western Yunnan and most areas in southern Hainan and Taiwan. The south-eastern areas in Guangdong showed high suitability and were supported by both individual and ensemble models (Figure 5;
Appendix Figure A2). In MaxEnt models, predictions based on dataset $\mathrm{B}$ were more liberal than those based on dataset $\mathrm{A}$ in Guangdong Province and other areas in southern China (Figure 5; Appendix Figure A2). In GBM and ensemble models, predictions of dataset $\mathrm{B}$ were more conservative than those based on dataset $\mathrm{A}$ (Figure 5; Appendix Figure A2). Transfers of models between native and coastal areas in southern China generally involved little extrapolation (see MOP results in Appendix Figure A3).

\section{Human risk assessment}

The human populations at risk of $S$. mansoni infection were estimated for Hong Kong, Dongguan, Huizhou and Shenzhen based on GBM predictions (Figure 5). Among the four cities, Hong Kong has the largest human population at risk of $S$. mansoni infection, i.e. there were about 40,000 humans at risk, whereas in Dongguan, Huizhou, and Shenzhen, there were less than 10,000 at risk in each city (Figure 6).

\section{Discussion}

Contrasting volume size, together with the overlaps of climate spaces occupied by native and introduced populations (Figure 2), suggest there are vast areas with suitable climate for $B$. straminea in China compared with those of the native Brazilian population. $B$. straminea is currently restricted to coastal areas in Guangdong Province but might have a large invasion potential in China. As can be seen in Figure 5, many suitable areas were identified in the coastal areas in Fujian, Hainan, Guangxi, Yunnan and Taiwan. High temperature is beneficial to snail spawning and may therefore create more suitable conditions for the reproduction and propagation of B. straminea (e.g., Scholte et al., 2012). Nonetheless, future climate warming will have complex effects on schistosomiasis transmission (Stensgaard et al., 2013; Pedersen et al., 2017; Zhu et al., 2017b), and it is conceivable that climate change will affect aquatic environments, and hence, alter the transmission and distribution of $S$. mansoni in this country.

Niche conservatism is much discussed with relevance to the ongoing climate change. For example, two major studies showed opposite results of niche conservatism during terrestrial plant inva-
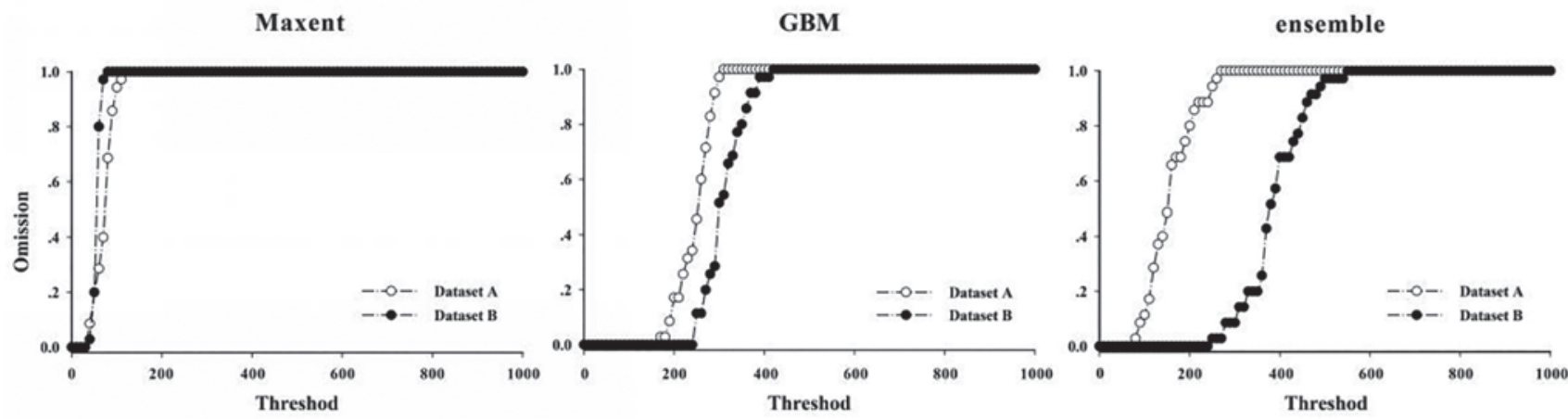

Figure 4. Omission errors of individual and consensus models based on the two datasets in anticipating introduced Chinese points. GBM, generalized boosted models. 
sion (Petitpierre et al., 2012; Atwater et al., 2017). In this study, the contradictory results of niche conservatism were observed based on the two datasets, with niche divergence supported in dataset A but not in dataset B. Thus, the environmental variables play an

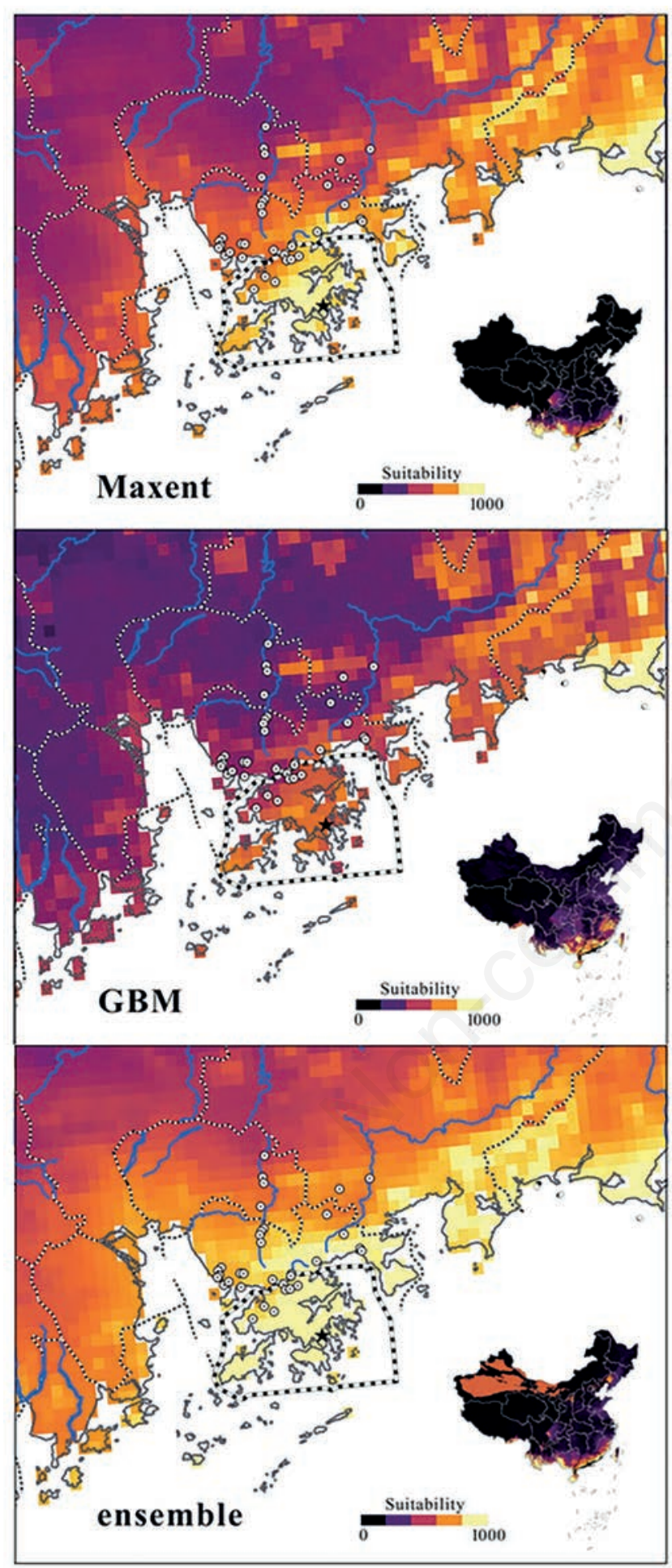

Figure 5. Potential distributions of Biomphalaria straminea in southern China. Native model transfers in China were based on three approaches (Maxent, generalized boosted model - GBM and ensemble) using dataset $B$. important role in niche conservatism evaluation (Zhu and Peterson, 2017). Niche conservatism represents the key assumption necessary for ENM application, especially when the models are used in an extrapolative manner (Owens et al., 2013). In this study, niche model predictions based on dataset B might be more reliable for $B$. straminea, showing highly suitable areas along coastal areas in Guangdong, Hainan, Taiwan as well as areas in south-western Yunnan Province (Figure 5).

Assessment of transmission risk and establishment of surveillance-response systems are critical to prevent $B$. straminea expansion. In China, the sampling sites of $B$. straminea were mostly in areas of fishing and farming, such as reservoirs, streams, and rivers (Figure 1). The main route of $B$. straminea expansion is from port city to mainland city (Pan et al., 1983). Among the four cities, many humans in Hong Kong reside within the suitable areas of $B$. straminea, where the highest probability of $S$. mansoni transmission may happen (Figure 4). Our predicted maps identified many suitable areas around its present distribution (Figure 5; Appendix Figure A2), the potential of $B$. straminea to disperse further in China is of great concern. The suitable map for B. straminea distribution identified in the present study will have implications in public health strategies, monitoring and guide fieldwork for earlier detection.

Contrary to former predictions, which either were based on introduced populations of a non-equilibrium state and identified suitable areas that clustered around observed points (Habib et al., 2016), or used single model that inherent uncertainty (Yang et al., 2018), our native models identified many suitable areas along southern coastal China and were consistent supported by multiple model approaches (Figure 5; Appendix Figure A2). These coastal cities are key areas of labour export, bearing high population mobility propagating pressure (Chen and Jiang, 2008), they deserve high attention regarding $S$. mansoni transmission or epidemics may happen, considering the synergistic effect of high climate suitability and propagation pressure (Simberloff, 2009).

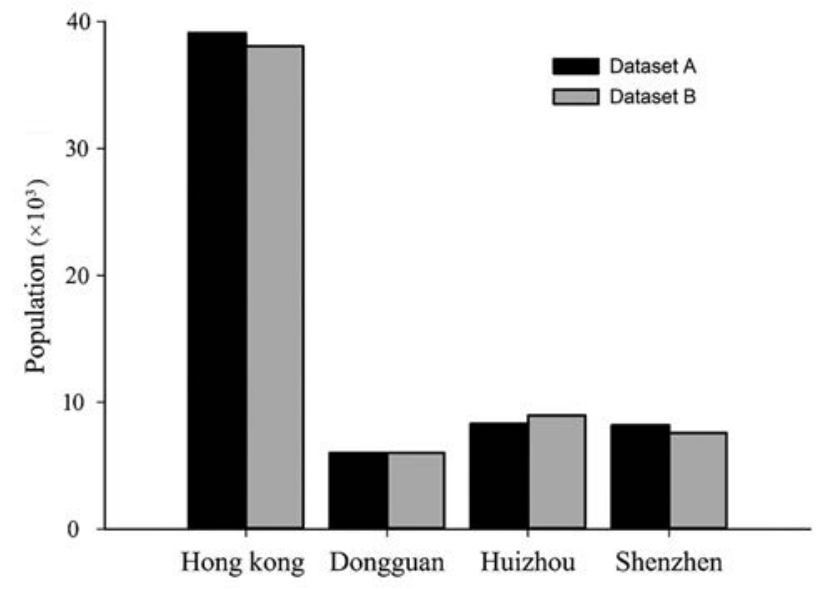

Figure 6. Human populations at risk of infection in four cities estimated by generalized boosted model. 


\section{Conclusions}

In this paper, we mapped the transmission potential of S. mansoni by predicting ecological dimensions and potential distributions of B. straminea using state-of-the-art ecological niche model approaches. Much suitable spaces were found to be unfilled in China, and might be available to $B$. straminea. Attention should be paid to $S$. mansoni epidemic may happen in coastal areas in Guangdong, Hainan, Taiwan, and areas in south-western Yunnan Provinces, considering high pressure of propagation, while future climate change may create even better conditions. Our results call for rigorous monitoring and surveillance in these identified areas in China.

\section{References}

Aelst S, Rousseeuw P, 2009. Minimum volume ellipsoid. Wires Comput Mol Sci 1:71-82.

Atwater DZ, Ervine C, Barney JN, 2017. Climatic niche shifts are common in introduced plants. Nat Ecol Evol 2:34-43.

Barve N, Barve V, Jiménez-Valverde A, Lira-Noriega A, Maher SP, Peterson AT, Soberón J, Villalobos F, 2011. The crucial role of the accessible area in ecological niche modeling and species distribution modeling. Ecol Model 222:1810-9.

Beans CM, Kilkenny FF, Galloway LF, 2012. Climate suitability and human influences combined explain the range expansion of an invasive horticultural plant. Biol Invasions 14:2067-78.

Broennimann O, Fitzpatrick MC, Pearman PB, Petitpierre B, Pellissier L, Yoccoz NG, Thuiller W, Fortin MJ, Randin C, Zimmermann NE, Graham CH, Guisan A, 2012. Measuring ecological niche overlap from occurrence and spatial environmental data. Global Ecol Biogeogr 21:481-97.

Chen ZY, Jiang HY, 2008. Propagule pressure - a mechanism for explaining biological invasion. J Yangtz (Nat Sci Edit) 5:7981.

Cola V, Broennimann O, Petitpierre B, Breiner FT, D'Amen M, Randin C, Engler R, Potter J, Pio D, Dubuis A, Pellissier L, Mateo RG, Hordijk W, Salamin N, Guisan A, 2017. Ecospat: An $\mathrm{R}$ package to support spatial analyses and modeling of species niches and distributions. Ecography 40:774-87.

Elith J, Graham CH, Anderson RP, Dudík M, Ferrier S, Guisan A, Hijmans RJ, Huettmann F, Leathwick JR, Lehmann A, Li J, Lohmann LG, Loiselle BA, Manion G, Moritz C, Nakamura M, Nakazawa Y, Overton JM, Peterson AT, Phillips SJ, Richardson K, Scachetti-Pereira R, Schapire RF, Soberón J, Williams S, Wisz MS, Zimmermann NE, 2006. Novel methods improve prediction of species' distributions from occurrence data. Ecography 29:129-51.

Escobar LE, Lira-Noriega A, Medina-Vogel G, Peterson AT, 2014. Potential for spread of the white-nose fungus (Pseudogymnoascus destructans) in the Americas: use of Maxent and NicheA to assure strict model transference. Geospat Health 9:221-9.

Fernandez M, 2002. Susceptibility of Brazil Biomphalaria straminea to in fection by 3 strains of Schistosoma mansoni. Mem I Oswaldo Cruz 97:59-66.

Habib MR, Guo YH, Lv S, Gu WB, Li XH, Zhou XN, 2016. Predicting the spatial distribution of Biomphalaria straminea, a potential intermediate host for Schistosoma mansoni, in China. Geospat Health 11:375-83.
Hijmans RJ, Cameron SE, Parra JL, Jones PG, Jarvis A, 2005. Very high resolution interpolated global terrestrial climate surfaces. Int J Climatol 25:1965-78.

Jiménez-Valverde A, 2012. Insights into the area under the receiver operating characteristic curve (AUC) as a discrimination measure in species distribution modelling. Global Ecol Biogeogr 21:498-507.

Kolar CS, Lodge DM, 2001. Progress in invasion biology: predicting invaders. Trends Ecol Evol 16:199-204.

Liu XH, Guo ZW, Ke ZW, Wang S, Li YM, 2011. Increasing potential risk of a global aquatic invader in Europe in contrast to other continents under future climate change. PLoS One 6:e18429.

Mack RN, Simberloff D, Lonsdale WM, Evans H, Clout M, Bazzaz FA, 2000. Biotic invasions: causes, epidemiology, global consequences, and control. Ecol Appl 10:689-710.

Muscarella R, Galante PJ, Soley-Guardia M, Boria RA, Kass JM, Uriarte M, Anderson RP, 2014. ENMeval: An R package for conducting spatially independent evaluations and estimating optimal model complexity for Maxent, ecological niche models. Methods Ecol Evol 5:1198-205.

Owens HL, Campbell LP, Dornak LL, Saupe EE, Barve N, Soberón J, Ingenloff K, Lira-Noriega A, Hensz CM, Myers CE, 2013. Constraints on interpretation of ecological niche models by limited environmental ranges on calibration areas. Ecol Model 263:10-8.

Pan SD, Chen PJ, Rong SM, 1983. Investigation on Biomphalaria straminea of Schistosoma mansoni intermediate host in Shenzhen areas. Guangdong Health Antiepidemic Data 7:70-6.

Pedersen UB, Karagiannis-Voules DA, Midzi N, Mduluza T, Mukaratirwa S, Fensholt R, Vennervald BJ, Kristensen TK, Vounatsou P, Stensgaard AS, 2017. Comparison of the spatial patterns of schistosomiasis in Zimbabwe at two points in time, spaced twenty-nine years apart: is climate variability of importance? Geospat Health 12:59-66.

Peterson AT, Papeş M, Soberón J, 2008. Rethinking receiver operating characteristic analysis applications in ecological niche modeling. Ecol Model 213:63-72.

Peterson AT, 2011. Ecological niche conservatism: a time structured review of evidence. J Biogeogr 38:817-28.

Peterson AT, Soberón J, 2012. Species distribution modeling and ecological niche modeling: Getting the concepts right. Nat Conservação 10:1-6.

Peterson AT, Moses LM, Bausch DG, 2014. Mapping transmission risk of lassa fever in West Africa: The importance of quality control, sampling bias, and error weighting. PLoS One 9:e100711.

Petitpierre B, Kueffer C, Broennimann O, Randin C, Daehler C, Guisan A, 2012. Climatic niche shifts are rare among terrestrial plant invaders. Science 335:1344-8.

Qiao HJ, Peterson AT, Campbell LP, Soberón J, Ji LQ, Escobar LE, 2015. NicheA: creating virtual species and ecological niches in multivariate environmental scenarios. Ecograph 39:805-13.

R Core Team, 2015. R: A language and environment for statistical computing. Vienna: R Foundation for Statistical Computing 14:12-21. Available from: www.R-project.org

Roura-Pascual N, Hui C, Ikeda T, Leday G, Richardson DM, Carpintero S, Espadalerf X, Gómez C, Guénard B, Hartley S, 2011. Relative roles of climatic suitability and anthropogenic influence in determining the pattern of spread in a global invader. Proc Natl Acad Sci USA 108:220-5. 
Scholte RGC, Carvalho OS, Malone JB, Utzinger J, Vounatsou P, 2012. Spatial distribution of Biomphalaria spp. the intermediate host snails of Schistosoma mansoni, in Brazil. Geospat Health 6:S95-S101.

Simberloff D, 2009. The role of propagule pressure in biological invasions. Ann Rev Ecol Evol S 40:81-102.

Soberón J, Nakamura M, 2009. Niches and distributional areas: concepts, methods, and assumptions. Proc Natl Acad Sci USA 106:19644-50.

Stensgaard AS, Utzinger J, Vounatsou P, Hürlimann E, Schur N, Saarnak CF, Simoonga C, Mubita P, Kabatereine NB, Tchuem Tchuenté LA, Rahbek C, Kristensen TK, 2013. Large-scale determinants of intestinal schistosomiasis and intermediate host snail distribution across Africa: does climate matter? Acta Trop 128:378-90.

Thuiller W, 2003. BIOMOD-optimizing predictions of species distributions and projecting potential future shifts under global change. Glob Change Biol 20:1353-62.

Yang Y, Cheng WT, Wu XY, Huang SY, Deng ZH, Xin Z, Yuan DJ, Yang Y, Wu ZD, Chen Y, 2018. Prediction of the potential global distribution for Biomphalaria straminea, an intermediate host for Schistosoma mansoni. PLoS Negl Trop Dis 12:e006548.

Veloz SD, 2009. Spatially autocorrelated sampling falsely inflates measures of accuracy for presence-only niche models. J Biogeogr 36:2290-9.

Zeng X, Yiu WC, Cheung KH, Yip HY, Nong W, He P, Yuan D, Rollinson D, Qiu JW, Fung MC, Wu Z, Hui JHL, 2017. Distribution and current infection status of Biomphalaria straminea in Hong Kong. Parasit Vectors 10:351-62.

Zhu GP, Fan JY, Wang ML, Li M, Qiao HJ, 2017a. The important of the shape of receiver operating characteristic (ROC) curve in ecological niche model evaluation - case study of Hlyphantria cunea. J Biosafety 26:184-90.

Zhu GP, Peterson AT, 2017. Do consensus models outperform individual models? Transferability evaluations of diverse approaches for an invasive moth. Biol Invasions 1-14.

Zhu GP, Fan JY, Peterson AT, 2017b. Schistosoma japonicum transmission risk maps at present and under climate change in mainland China. PLoS Negl Trop Dis 11:e0006021. 